Paediatrica Indonesiana $15: 273$ - 285. Nov. - Dec. 1975. 273

From the Gezondheid Organisatie $T N O \&$ 's Gravenhage, Nederland.

\title{
Epidemiological Trends of Tuberculosis in Low and High Prevalence Countries.
}

by

\section{$M \cdot A, B L E I K E R$}

\begin{abstract}
The study of the epidemiology of tuberculosis and the need for a rational approach to the problem of tuberculosis control require a sound knowledge of the risk of transmission of tuberculosis infection from host to host. This knowledge is required today both in countries with a high prevalence and in those with a low prevalence of the disease.

The risk of transmission of tuberculosis infection in a given community during a particular period of time is most reliably expressed numerically in terms of a series of average annual inlection risks in successive calender years.

The infection risk indicates the proportion of the population which will be primarily infected, or reinfected, with tubercle bacilli in the course of one year. The risk is usually expressed us a percentage or as a rate. The annual trend (decrease) in the risk of infection is even more important than the actual level of this risk.

Tuberculin surveys in schoolshildren, which are repeated, say every five years, in the same area provide us with the necessary information to make estimates for the annual infection risk. In areas where infections with the socalled atypical mycobacteriae are frequent, simultaneous testing with two "Sensitins", one prepared from human tubercle bacilli and one from atypical mycobacteriae will be of value for estiniating the proportion of those infected with virulent tacilli and those infected with other mycobacteriae.
\end{abstract}

*) Invited paper at the Third National Indonesian Paodiatric Congress, Surabaya, July 1 - 6, 1974.

Received 11th. September 1974. 


\section{Introduction}

In the present stage of development where tuberculosis mortality has lost its istatistical significance because of effective chemotherapy, it is generally recognized that the most relliable measure of the extent of the tuberculosis problem in a population is the "annual tuberculosis infection rate" or incidence of infection. In countries where infection with the bovine type of tubercle bacilli no longer exists, as is the case in the majority of dow prevalence countries, the annuall infection rate expresses that proportion of the population under study which will be primarilly infected, or reinfected (in those who have been previously infected) with tubercle bacilli from a human source in the course of one year.

The annual tuberculosis infection rate is also the best measure for following the trend of the tuberculosis problem in a given populiation and for evaluating the total effects of organized efforts to control tuberculosis.

The annuall tuberculosis infection rate is derived from the results of tuberculiun testing. A technique for converting information on prevallence of infection into a smooth series of annual rates of tuberculosis infection has been developed recentlly by TS$R U$ and published in a comprehensive report in 1969 (TSRU, 1969). To obtain raliable estimates of the annuall tuberculosils infection rates and their changes in a particular period, several tuberculin surveys are required ait intervals, each in a representative sample of non-BCG vaccinated subjects of the same age, tested by the same technique.

Dependable information on annual tuberculosis infection rates, and on the trends in the rates in recent years, enables a low prevalence country to assess the rellevance of current ituberculosis control measures in a rapidlly changing situation, and to plan for the eventual eradication of the disease. A considierable advantage of this epidemiological index is that it is not linked with any part of a tuberculosis programme, such as diagnosis and reporting of cases.

The approach used in TSRU for estimating the average annual risks of tubercullous infection in the Netherlands is complicated, partly because nothing was previously known about the way jin which the risk of infection was changing and this had to be assessed carefully, and partly because it was jesirable, in the process, to make comprehensive use of the extensive prevalence data available in the Netherlands. However, for routine itubercullosils control a simple method of estimating the annual tuberculosis infection rate is described in the short section VII of the same report. 
I am going to describe once more how the annual tuberculosis infection rates can be estimated easily, because a number of people who have recently visited the International Tuberculosis Surveillance Centre, to which I will refer later, knew about the TSRU report on the transmission of infection but found the publication too technical.

There are:

Two steps in assessing the annual tuberculosis infection rates.

a. Estimation of the percentage decrease an the annual risk of infection using Appendix Table $\mathrm{C}$ of TSRU report no. 1.

Table 1 shows a part of this table. The percentage decrease in the annual tuberculosis infection rates can be estimated if two or more prevalence figures are available for subjects of the same age. If the prevalence of infection in children aged (say) 10 years was, for instance, $5.5 \%$ in 1966 , and $2.5 \%$ in 1972 , entry 80 in the table is divided by 6 years (from 1966 to 1972), to give the approximate annual percentage decrease, which is about $13 \%$. The approximate annual percentage decrease is needed for use in Appendix Table $B$.

b. Using the estimate of the percentage decrease, Appendix Table B of the same report provides direct assessments of the risk of tuberculous infection in two calendar years, namely the year in which the prevalence of tuberculous infection was determined, and a few years earlier. In the above mentioned case one consulits Appendix Table B for children aged 10 years, last column (13\% annual decrease in risk of infection each year). The table indicates the following annual tuberculosis infection rates:

1966 : $0.25 \%$ (and in 1956 : $0.92 \%$ )

$1972: 0.11 \%$ (and in 1962 : $0.41 \%)$.

Trend in the risk of tuberculous infection with age

It is widely believed that the risk of tuberculous infection may be greater among adolescents and young adults than among children or older people. A small (though non-silgnifi cant) trend in infection risk with age (about $2 \frac{1}{2} \%$ for each year of age during the period 1945 to 1966) was observed in TSRU report no. 1.

Funther studies carried out by Sutherland and Fayers and by Waaler provide evidence of a consistent tendency for the risk of tuberculous infection to increase with age in a number of countries, both developed and developing. More detailed analy. ses are being made by Sutherland and Fayers on the data from a number of countries. The finding that the risk of infection is associated 
with age in several countries sug. gests that a simillar association may also exists in other countries. It is difficult to assess how the risk vanies with age, but as already indicated, the calendar trend in the risk, eliminating a possible effect of age, is readily estimated from the results of two or more tuberculin surveys on representative samples of the popullation of the same age, (say) every 5 to 7 years.

The risk of tuberculous infection and its decrease in low prevalence countries

Some of you know the curve shown on Figure 1 referring to the level and the trend of the risk of tuberculous infection in the Netherlands over a long period. The risk of tuberculous infection in that country is, at present, very low indeed - of the order of 0.3 per thousand in 1973 - and it has been decreasing steadily by about $14 \%$ annually since 1941 .

We now also know more about the risk of tuberculous infection in other developed countries (see figure 2). Junker has recently published estimates of the risk of tuberculous infection in Vienna (Austria) for the first 50 years of this century. From his curve, we have plotted the part between the years 1922 and 1948 based on the results of tubercullin testing carried out in 1927 (nearly 14,000 children aged $61 / 2$ years) and in 1948 (nearly 8,000 children aged 6-7 years). The esti- mated risk of tuberculous infection was nearly $6 \%$ in 1922 and about $2.2 \%$ in 1948. Because of the lack of reliable information on the prevalence of tuberculous infection in children of that age between 1927 and 1948, the hypothetical straight line of the risk presented in the figure might have differed from the real trend of the risk. On the other hand, estimates of the risk of tuberculous infection for $1922(6 \%)$ and $1948(2.2 \%)$ are reliable. It can be concluded therefore, that there has been a considerable decrease in the risk of infection during the period under study.

Sutherland et al., estimated the risk of infection for Prague (Csechoslovakia), also for the period 1922 to 1948 . Estimates are derived from the results of tuberculin testing of 22,000 children aged 6-10 years in 1926 and about 78,000 children aged 5-15 years tested in the ITC in 1948. (TSRU report no 2). The risk of tuberculous infection in Prague was about $8 \%$ in 1922 and about $2 \frac{1}{2} \%$ in 1948.

The results from the Netherlands, Vienna and Prague show (a) that the risk of tuberculous infection was high in those three parts of Europe before World War II, and (b) that the risk decreased, on average, by about $4 \%$ to $5 \%$ each year. There are very few other historical data available that are fully representative for any of the European countri- 
es. It is therefore useful to look more to the present and to the near future.

Neumann carried out a tuberculin survey of about 500 children in Stuttgart (West Germany) with the WHO standand tuberculin test in 1965. The second tuberculin survey of nearly 2,000 school-children aged 7 to 15 years was made in June 1971, in co-operaition with ITSC. Schools included in the 1965 survey were also included in the 1971 survey; in addition, a number of other schools in Stuitgart participated in the latter survey. The analysis of the material showed that the present risk of tuberculous infection in Situitgart is very low indeed - about 0.3 per thousand. The decrease in the risk of infection with calendar year was nearly $15 \%$. The levels of the rates and the decrease in the annual risk of infection are of the same magnitude as was found in the Netherlands.

Waaler, Galtung and Mordal estimated the risk of infection in Norway. Their estimates were derived from the results of tuberculin testing in school-children carried out on a national basis during the last two decades. The materilal is very reliable. Their estimates are very close to those in the Netherlands, both with regard to the level of the risk of infection and its downward trend. A full report will appear soon.
The TSRU analyses of the material from Saskaitchewan (Canada) allso show that the sintuation in Canada is similar to that in the Netherlands and other low prevalence countries. The material from Saskatchewan is very extensive and is reliable; about 140,000 tuberculin tests in subjects of all ages were made annually during the period 1954 to 1970 .

Lotte is going to present the results of estimates of the risk of infection in France. A representative tuberculin survey is being made in Britain and we hope that our British colleagues will soon publish a full report of estimates of the risk of infection and its trend. Preliminary results, derived from the data on tuberculin testing completed during the first year of the study, show that the present risk of infecion in Britain is also very low.

Our team has recently made a tuberculin survey in Sandinia (Italy). The prevalence of indurations of $10 \mathrm{~mm}$ or more to 2 TU PPD Ri 23 in schoolchildren aged 6 to 14 years was $3.0 \%$. This correspond's to a rusk of infection of less than 3 per thousand.

We think that these resulits show two facts :

1. In the majority of technically developed countries there is, at present, a low or a very low risk of tuberculous infection. It is very probable that in the majori- 
ty of developed countries the present risk of tuberculous infection is below 5 per thousand; in most of these countries the rate may be of the order of 1 to 3 per thousand; and in a few of those countries the annual risk of tuberculous infection is even less than 1 per thousand.

2. The risk of tuberculous infection contimues to decrease, in developed countries, by about $10 \%$ or more each year. Thus even these smail risks may halve themselves every 5 to 7 years.

A different situation is seen in countries with high tuberculossis-prevalence-figures. The risk of infection is not only high, but what is much more important, the decrease of the risk is very small or not seen at all.

Stott and collaborators have analyzed thoroughly the risk of infectiron in Uganda. As a first base lime for the estimate of the risk of infection the results of a tuberculin survey made in 1958 through the WHO were used. The results of the survey made in 1970-1971 were used as second information. The same 10 areas as in this study (1958) were visited for tuberculin-testing.

The tuberculosis infection rate for 1958 was $2.6 \%$ and for 1970 it was 2.3\%. The slight decrease in the infection rate was smaller than $1 \%$ per year and this is not statistically significant.

ITSC analyzed the data concerning the prevalence of infection in one of the North-Africa countries for this same period 1950 - 1971. The first investigations in children in this country were made in connection with the BCG-campaign of ITC in 1948. The ITSC-team tested in 1971 schoolchildren in four areas, in which the coverage of the BCG-vaccination in 1948 had been low.

The results are given for two areas in which the infection risk in 1950 was almost the same: $3,9 \%$ and $3,1 \%$. The decrease in the risk of infection however was substantially different, in one of the areas (ur. ban) it declined to $1 \%$ and in the other (rural) it declined only to $2.3 \%$.

We have a number of tuberculosis infection risks from various high prevalence countries, but unfortunam tely we do not yet have a trend. The risks however, are high, e.g. in $\mathrm{Za}$ ire more than $2 \%$ and in the western part of Java more than $3 \%$. The risk proved to be lower in e.g. Suriname, $1 \%$ and in Venezuela $0,8 \%$.

The risk itself and its downward trend decrease much slower in these high prevalence countries.

We are of the opinion that in these high prevalence countriles steps shouild be taken in the fight against 
tuberculosis, which enable and ensure a rapid decline of the risk of infection for tuberculosis.

Why is the annual tubercubosis infection rate not used on a larger scale?

There are, in our opinion, several reasons and explanations for this:

a. The method has been introduced only recently. TSRU report no. 1 appeared in 1969 and it is understandable that after the introduction of a new epidemiological index some time will elapse befo$r e$ it is generally used.

b. As already mentioned, the TSRU approach in report no. 1 may seem too complicated to a number of policy makers. However, it has been shown that the method is simple.

c. In a number of countries the method cannot be used at present because of compulsory BCG vaccination of newborns. Unless a representative sample of new. born children is selected and BCG vaccination is deferred in this group of children for severa] years, in order to assess the development of tuberculin sensitivity during this period, the annual infection rate cannot be esstimated reliably.
However, it is possible, in these developed countries, to arrange regular tuberculin testing of this group of children for a number of years and to give chemoprophylaxis to those who have acquired tuberculous infection. In fact, it is essential for those countries to discover the right polint at which to stop mass BCG vaccination, namely when the harm from BCG vaccination (in terms of serious complications, such as suppurative lymphadienitis, lupus vulgaris, fatal BCGitis, loss of tuberculin allergy, etc.) is greater than the benefit from BCG vaccination. The risks of tuberculous infection shown previously indicate that in some countries such a point has been reached already or will very soon be reached.

d. In countries where there is a high prevalence of "non-specific" tuberculin sensittivity, the efficiency of the tuberculin tesit as a measure of tuberculous infection is somewhat reduced.

The currently available techniques, however, make it possible to determine with sufficient accuracy the proportion of persons infected with tubercle bacilli. 
TABLE 1 : Decreases in infection risk correspawding to various percontages infected by the same age at two different surveys (tsru).

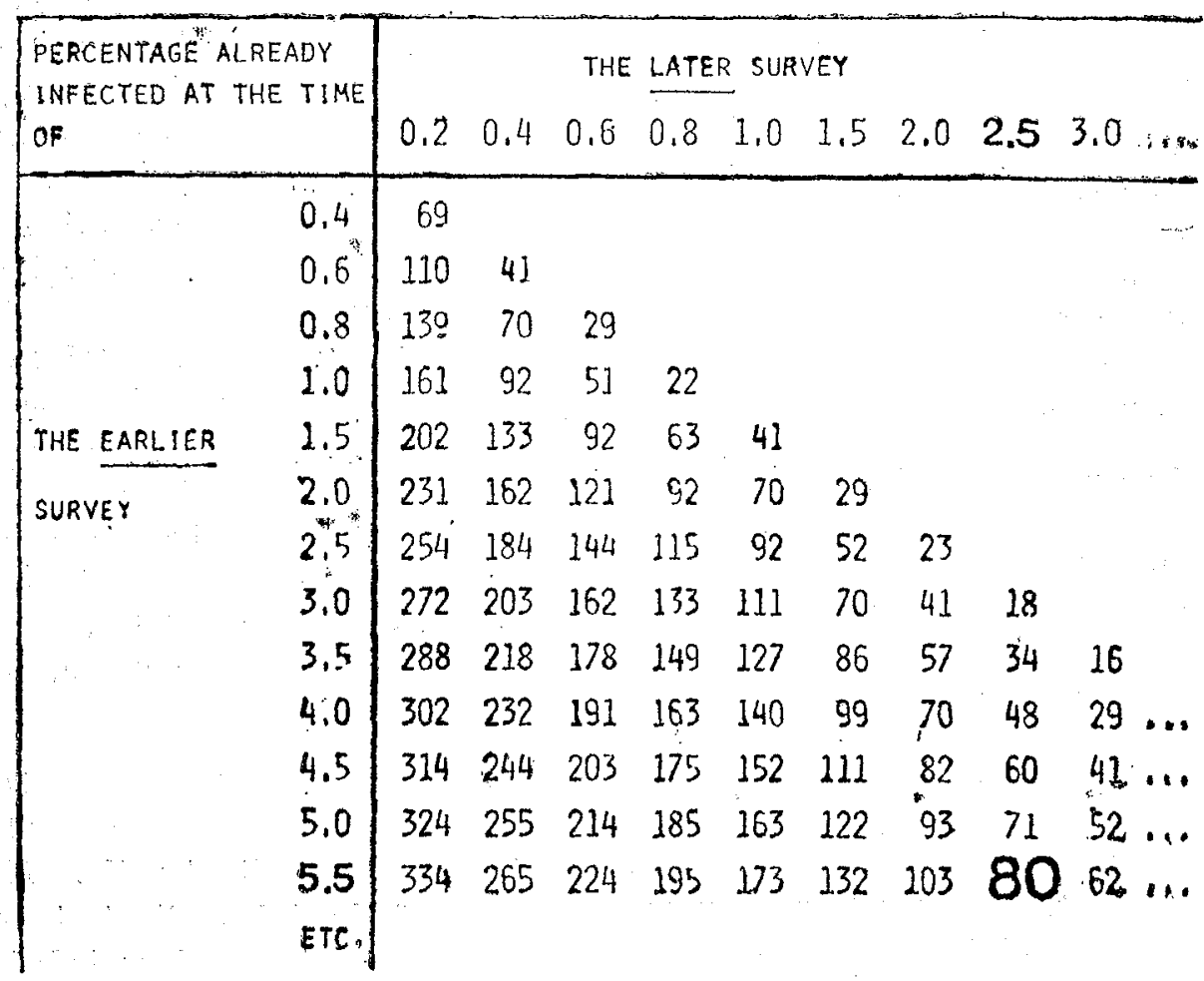

Divide the entry in the table by the interval in years between the surveys to obiain the approximate annual percentage decrease for use in app. table $b$. 
TABLE 2 : Ammual percentage risks of tuberculous infection corresponding to the percentage already infected by the age of 10 years (tsru).

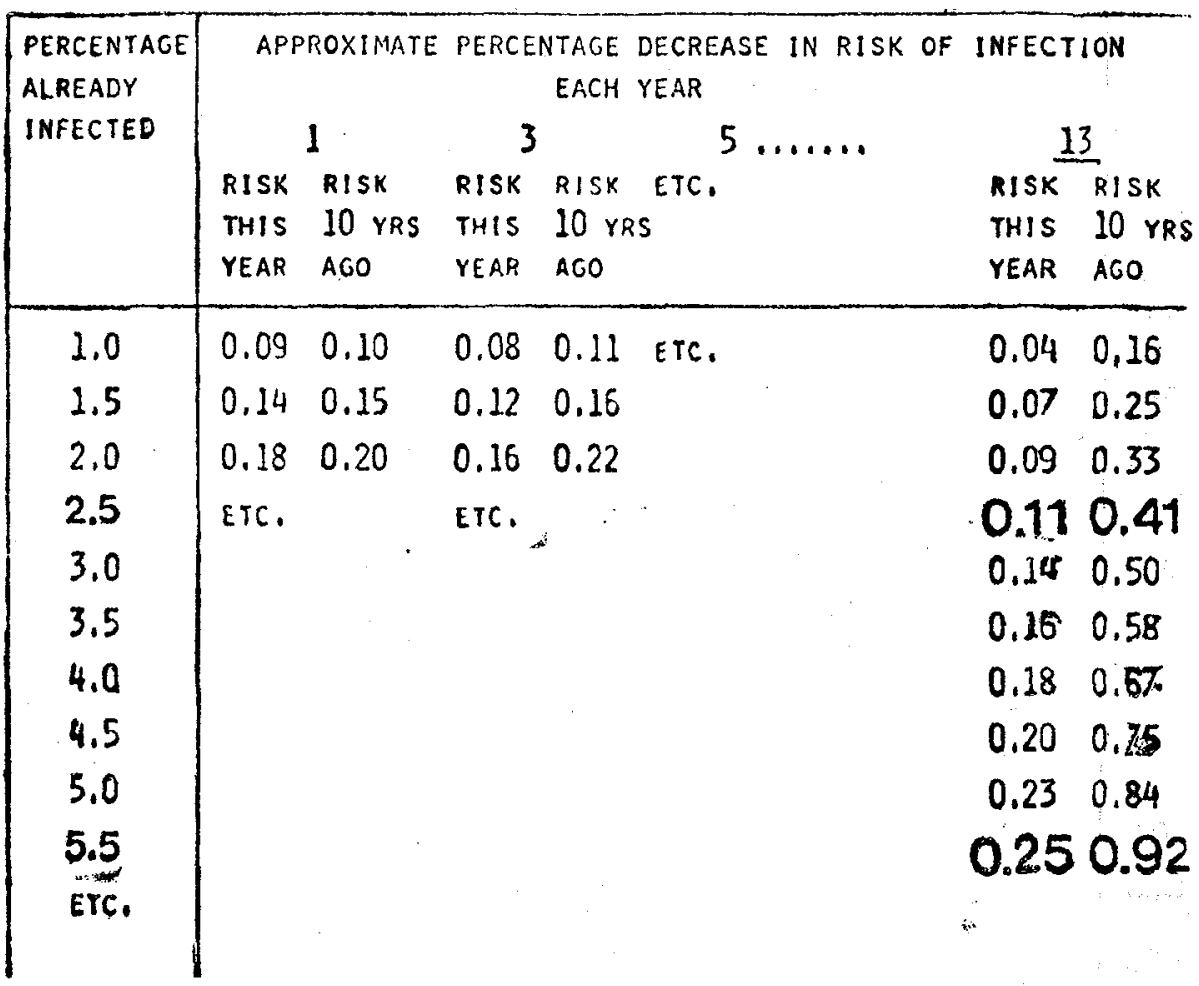


ESTIMATED ANNUAL RISK OF TUBERCULOUS INFECTION

$1950 \_1971$

\section{Risk of tb}

infection $(\%)$

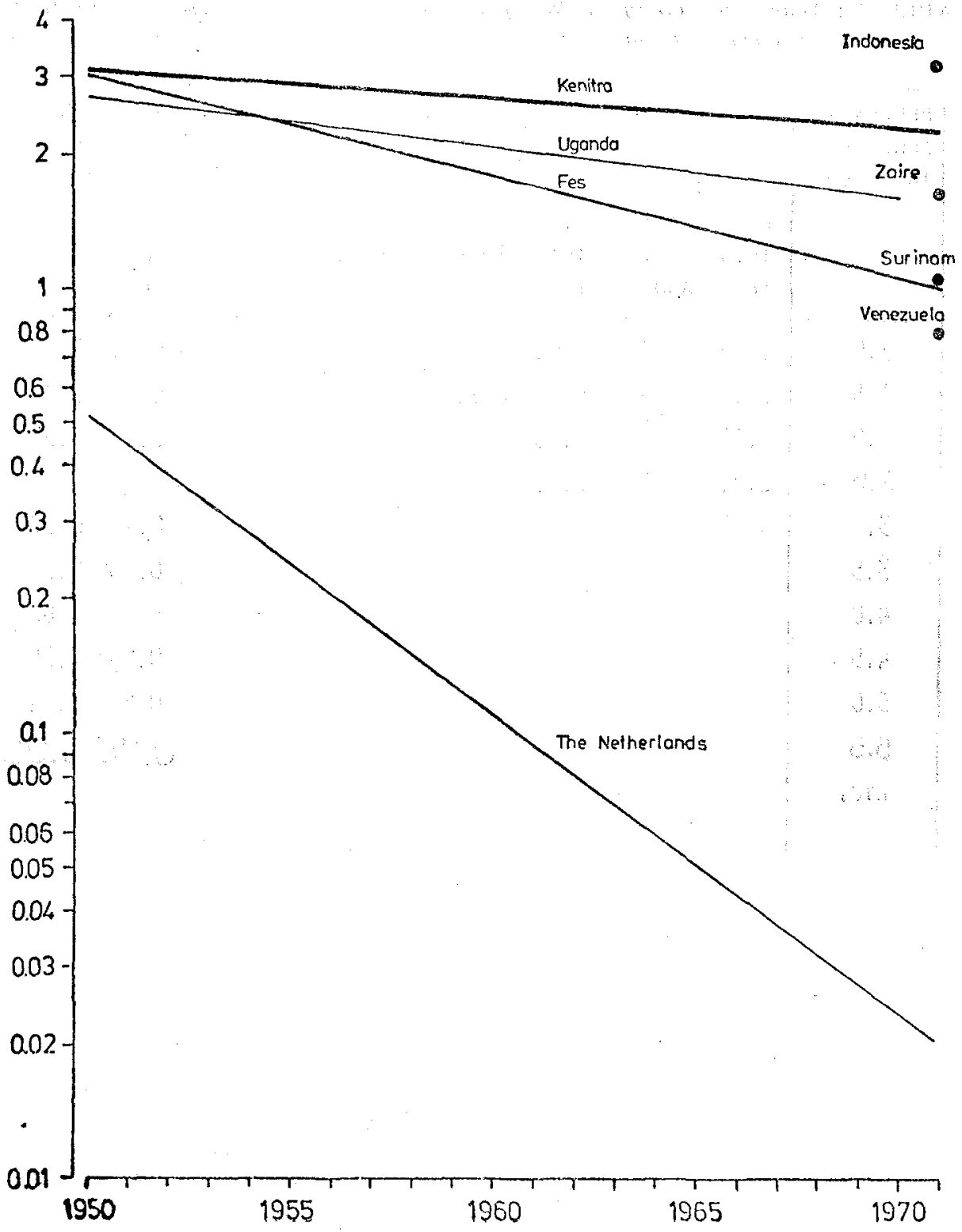


ANNUAL RISK OF TUBERCULOUS INFECTION (\%)

THE NETHERLANDS, $1910-1970$

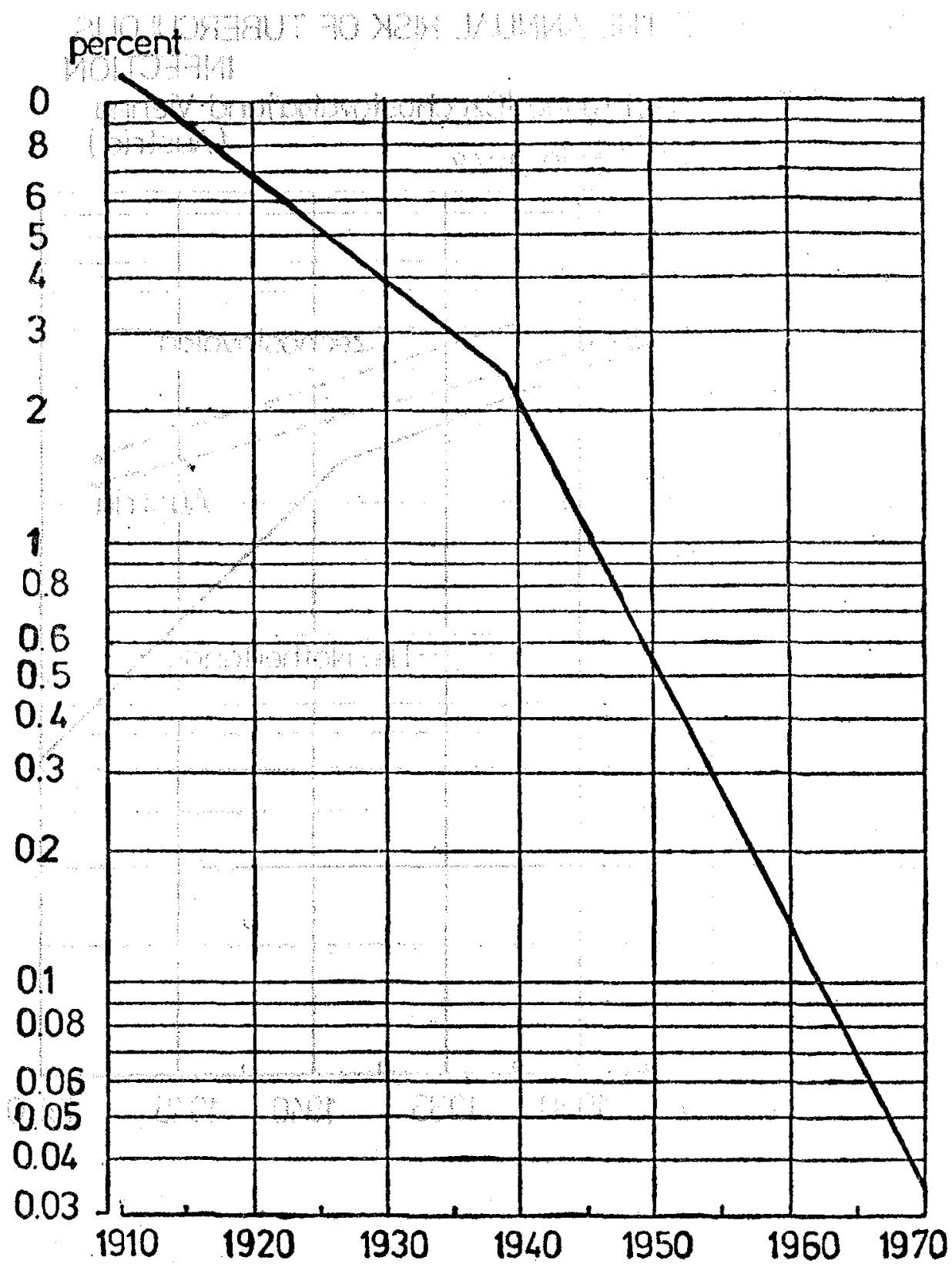


ESTIMATES OF THE ANNUAL RISK OF TUBERCULOUS INFECTION The Netherlands. Prague (Czechoslovaka) and Vienna

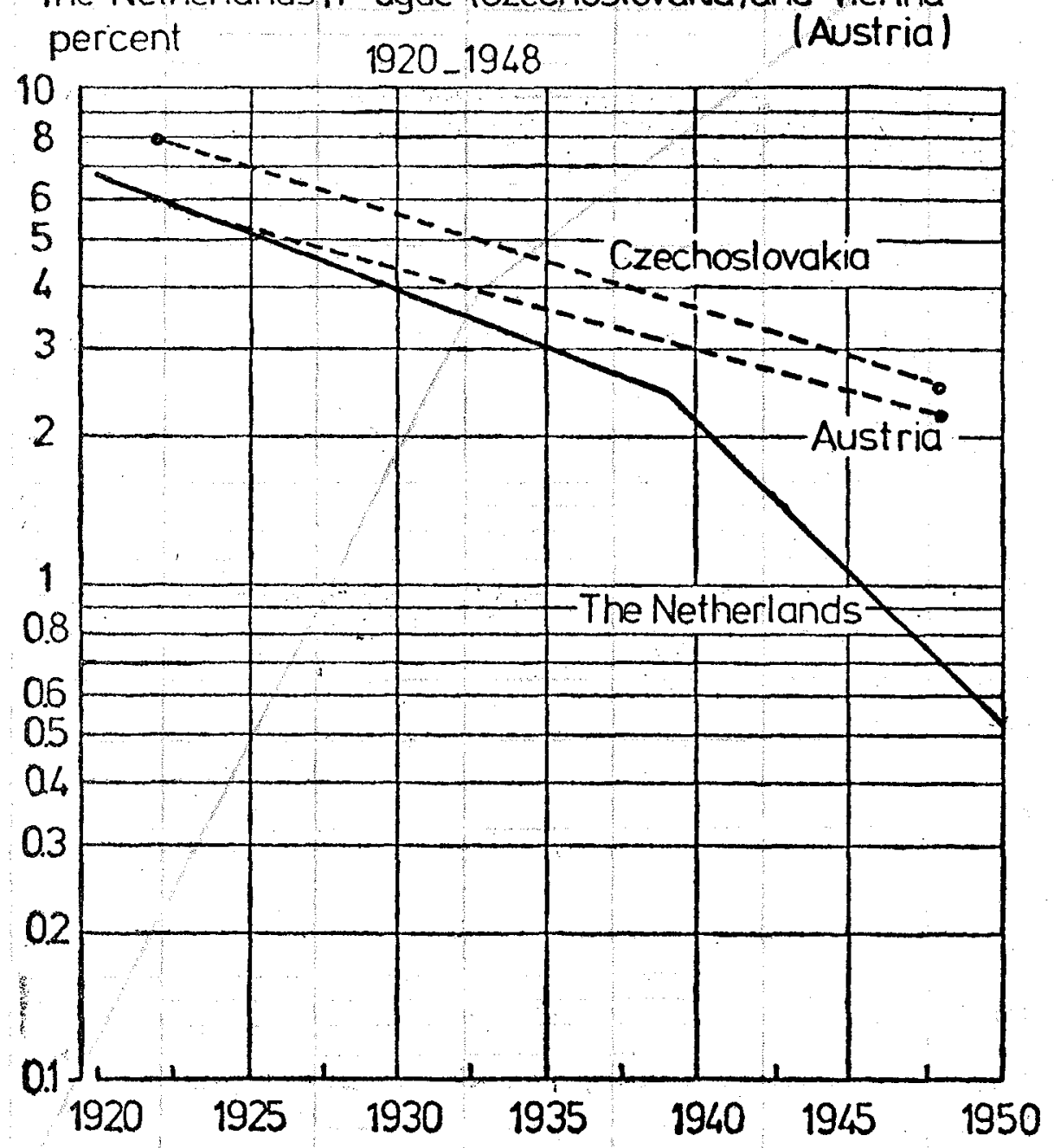


LOW PREVALENCE COUNTRIES. 1955 _ 1972

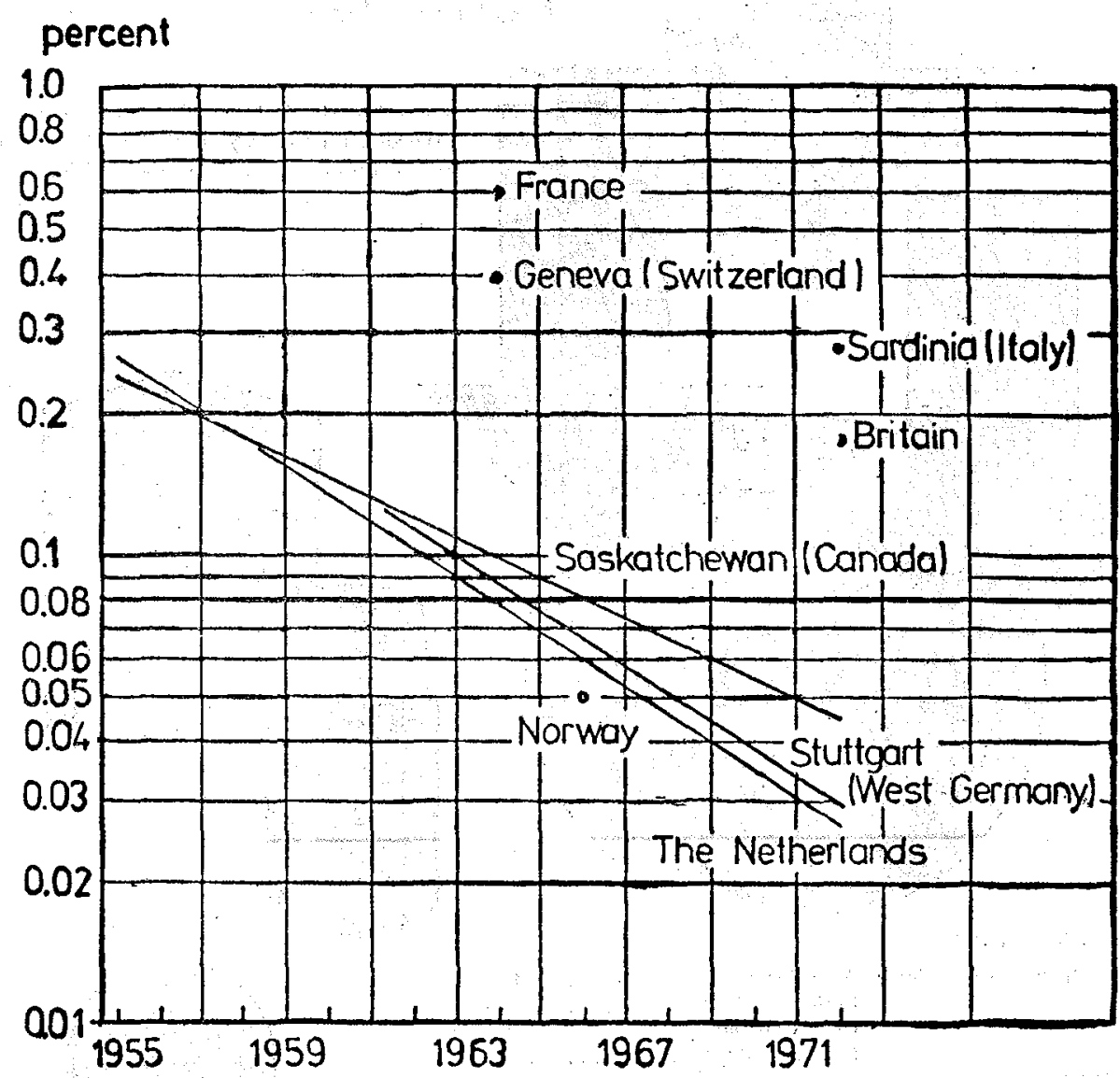

